\title{
Backscattering analysis of impedance loaded wire antenna for passive RFID applications
}

\author{
Chien-Chang Huang ${ }^{\text {a) }}$ and Kai-Wei Ku \\ Department of Communication Engineering, \\ Yuan Ze University, Taoyuan, Taiwan \\ a)cch@saturn.yzu.edu.tw
}

\begin{abstract}
The dynamic backscattering analysis of impedance loaded wire antennas for passive RFID applications is presented where the two loading impedances represent the corresponding logic states in the transmitting data of RFID tags. The analysis is based on the equivalent circuit of receiving antennas from moment method and the timevarying load calculation from the conversion matrix technique in the frequency domain. Simulation studies on $915 \mathrm{MHz}$ with resistive or capacitive loads are shown where the reactive load indicates more apparent discrimination for the two logic states, thereby improving the receiving sensitivity of RFID readers. The analysis methods and results can provide useful information for antenna and IC designs of RFID tags.
\end{abstract}

Keywords: RFID, backscattering modulation, impedance loaded antenna

Classification: Microwave and millimeter wave devices, circuits, and systems

\section{References}

[1] R. Want, "An introduction to RFID technology," IEEE Pervasive Computing, vol. 5, pp. 25-33, Jan.-March 2006.

[2] P. V. Nikitin and K. V. S. Rao, "Theory and measurement of backscattering from RFID tags," IEEE Antennas Propaga. Mag., vol. 48, pp. 212218, Dec. 2006.

[3] R. E. Collin, "Limitations of the Thevenin and Norton equivalent circuits for a receiving antenna," IEEE Trans. Antennas Propaga., vol. AP-45, pp. 119-124, April 2003.

[4] W. Geyi, "Derivation of equivalent circuits for receiving antenna," IEEE Trans. Antennas Propaga., vol. AP-52, pp. 1620-1623, June 2004.

[5] R. Janaswamy and S.-W. Lee, "Scattering from dipoles loaded with diodes," IEEE Trans. Antennas Propaga., vol. AP-36, pp. 1649-1651, Nov. 1988.

[6] C.-C. Huang and T.-H. Chu, "Analysis of wire scatters with nonlinear or time harmonic loads in the frequency domain," IEEE Trans. Antennas Propaga., vol. AP-41, pp. 25-30, Jan. 1993. 
[7] K. Penttila, M. Keskilammi, L. Sydanheimo, and M. Kivikoski, "Radar cross-section analysis for passive RFID systems," IEE Proc. Microwaves Antennas Propaga., vol. 153, pp. 103-109, Feb. 2006.

[8] W. L. Stutzman and G. A. Thiele, Antenna Theory and Design, 2nd Edition, John Wiley \& Sons, Inc., NY, 1998.

[9] R. F. Harrington, Field Computation by Momoent Methods, Macmillan, NY, 1968.

[10] EPC Radio-Frequency Identity Protocols Class-1 Generation-2 UHF RFID Protocol for Communications at $860 \mathrm{MHz}-960 \mathrm{MHz}$, Version 1.2.0, EPC Global Inc., 2008.

[11] B. Razavi, Design of Analog CMOS Integrated Circuits, McGRAW-HILL, NY, 2001.

\section{Introduction}

The passive RFID system [1] utilizes the backscattering of the RFID tags with different loaded states to transmit the short data stored in the tags. The backscattering properties are determined by the antenna structures, called the structural mode, and the loaded impedances, called the antenna mode [2] where the structural mode is due to the induced current of the antenna from the incident field and the antenna mode comes from the impedance mismatch between the antenna and the load. The backscattering problem of the passive RFID tags then can be simplified to the equivalent circuits in Thevenin or Norton configurations [3, 4] with time-varying loads. There are some research results, though not in the applications of RFIDs, on the loaded antennas with linear [5], nonlinear or time-varying [6] characteristics. The specific RFID backscattering studies by radar-cross-section can also be found [7]. However, these results are in the static case with short/open or resistive load environments. The theoretical analysis of RFID dynamic operations with resistive/reactive loads has not been addressed in literatures.

This letter presents an analysis approach for the dynamic backscattering operations of RFID tags based on the equivalent circuit of receiving antennas from moment method and the conversion matrix method [6] for the resistive/reactive time-varying loads as shown in Fig. 1(a). The incident field is assumed as a uniform plane wave. Both of the antenna part and the load part are treated in the frequency domain, while the backscattering waveforms are calculated by the simple Fourier series to observe the modulation characteristics. A linear wire antenna with resistive or capacitive load changed in two states for resistances or capacitances are demonstrated to show the validity of the developed approach.

\section{Formulation}

The Pocklington's integral equation of a linear wire antenna is described as [8]

$$
\frac{1}{j \omega \varepsilon_{0}} \int_{-L / 2}^{L / 2} I\left(x^{\prime}\right)\left[\frac{\partial^{2} \psi\left(x, x^{\prime}\right)}{\partial x^{2}}+k^{2} \psi\left(x, x^{\prime}\right)\right] d x^{\prime}=E_{x}^{i n c}(x),
$$




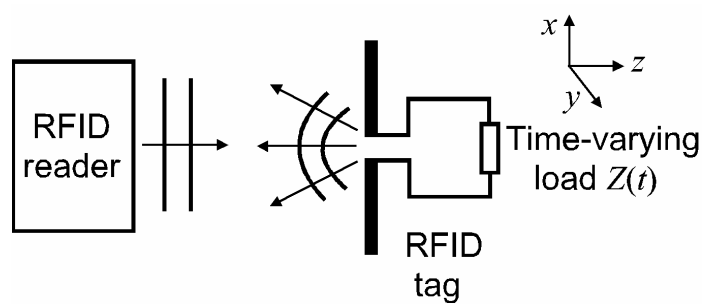

(a)

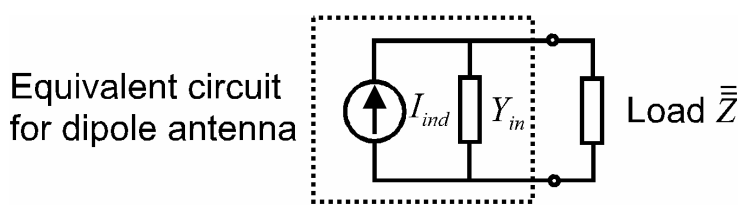

(b)

Fig. 1. (a) Illustration of RFID system operation and (b) equivalent circuit of RFID tag.

where $k^{2}=\omega^{2} \mu_{0} \varepsilon_{0}$ is the free-space wave number, $I\left(x^{\prime}\right)$ is the unknown current distribution in the wire antenna with length $L$ and radius $a ; \psi\left(x, x^{\prime}\right)=$ $e^{-j k r} /(4 \pi r)$ is the free-space Green function, $r=\sqrt{\left(x-x^{\prime}\right)^{2}+a^{2}}$, and $E_{x}^{i n c}(x)$ is the incident electric field in the same polariztion direction with the linear wire antenna.

With the utilization of the basis function expansion for the unknown current and the testing function for the equation (known as the momoent method), (1) can be reformed as a linear matrix equation where the solution is the induced current source $I_{\text {ind }}$ of the equivalent circuit shown in Fig. 1 (b). The antenna input admittance $Y_{i n}$ is deduced by setting the incident field as the impressed field in the delta-gap for unit-voltage applied [8].

If the RFID operating frequency is $\omega_{0}$ and the change-rate of the loaded resistor for backscattering is $\omega_{p}$, the $i-v$ relationship of the time-varying resistor can be described by the series representation as [6]

$$
\sum_{n=-\infty}^{\infty} V_{n} e^{j\left(\omega_{0}+n \omega_{p}\right) t}=\sum_{n=-\infty}^{\infty} \sum_{m=-\infty}^{\infty} R_{n} I_{m} e^{j\left[\omega_{0}+(n+m) \omega_{p}\right] t}
$$

where $R_{n}$ is the Fourier coefficient of the waveform for the time-varying resistor. The sereis is truncated from $-M$ to $M$, and (2) is expressed as a matrix equation as [6]

$$
\bar{V}=\overline{\bar{R}} \cdot \bar{I},
$$

where $\overline{\bar{R}}$ in order of $(2 M+1) \times(2 M+1)$ is composed with the Fourier coefficients and is further defined as the conversion matrix [6]. On the other hand, the time-varying capacitive load can be treated as well except an additional frequency term as $[6]$

$$
\bar{I}=j \overline{\bar{\Omega}} \overline{\bar{C}} \cdot \bar{V} .
$$

Note that $\overline{\bar{\Omega}}$ is a diagonal matrix whose elements are the frequencies in (2). Other time-varying load circuits including the inductors are formulated in the similar way - from the scalar relations to the matrix ones. The final terminal 


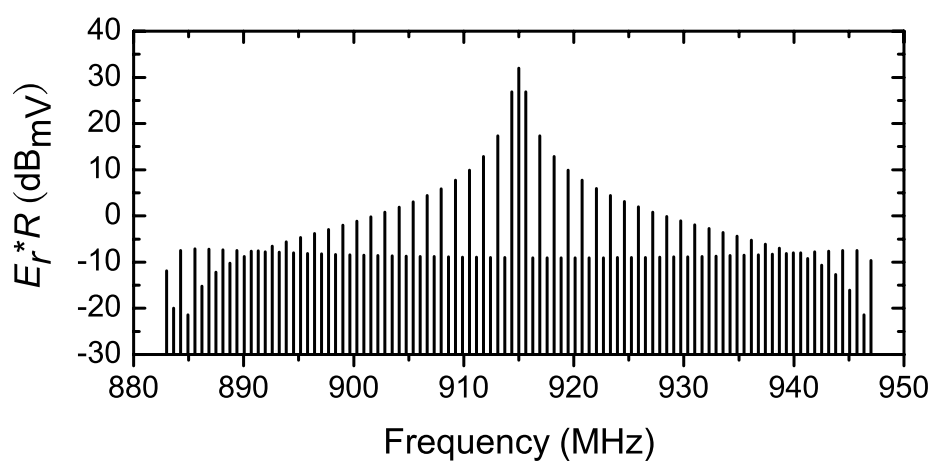

(a)

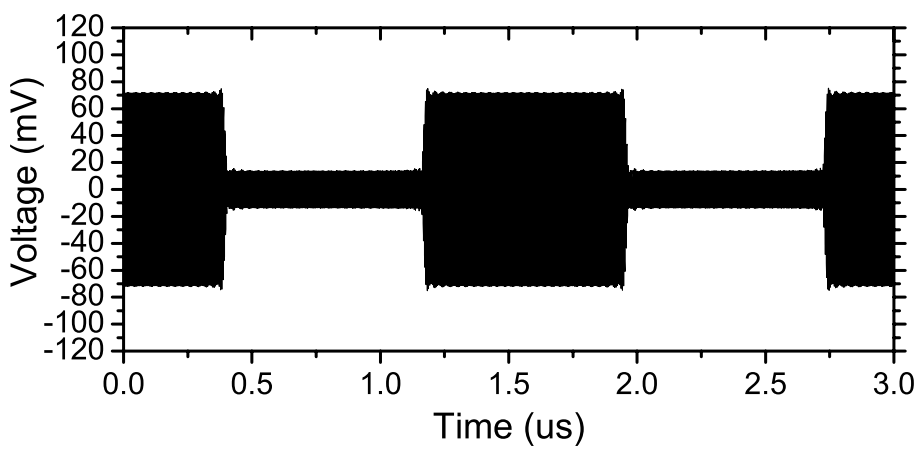

(b)

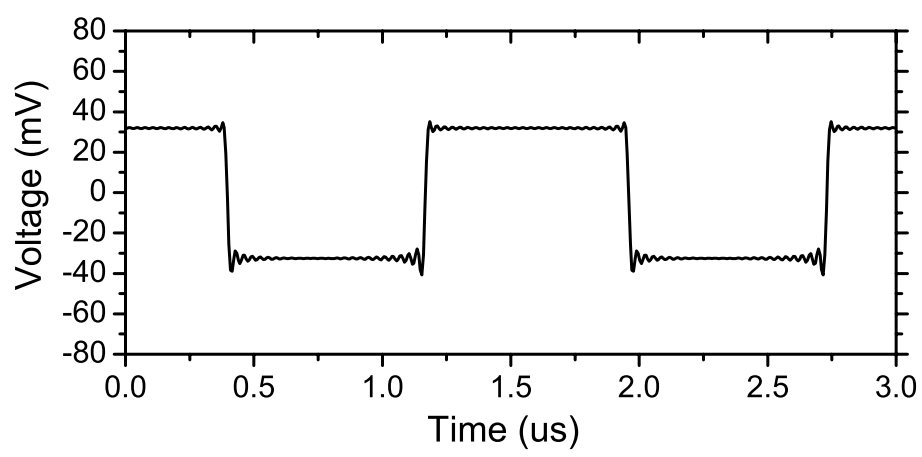

(c)

Fig. 2. Scattered field of linear wire antenna with resistive time-varying load represented in (a) spectrum, (b) time-domain waveform, and (c) coherent demodulation waveform.

voltage $V_{i n}$ of the linear wire antenna then is combined with the antenna input impedance and the loaded impedance expressed in matrix forms for various spectral components.

For the backscattering field, it is contributed by two parts: one is the antenna structure and the other is the loaded impedance. The far field expression of the backscattering for the time-varying loaded antenna can be derived by reciprocity $[9]$ as

$$
E_{r}=\frac{\omega \mu_{0}}{4 \pi R}\left\{\left[V^{r x}\right]^{T}[Y]\left[V^{t x}\right]+\left[V^{r x}\right]^{T}[Y]\left[V^{s r c}\right]\right\},
$$

where $[Y]$ is the admittance matirx from the moment method solution of the antenna, $\left[V^{t x}\right]$ and $\left[V^{r x}\right]$ are the excitation vector and the measurement 


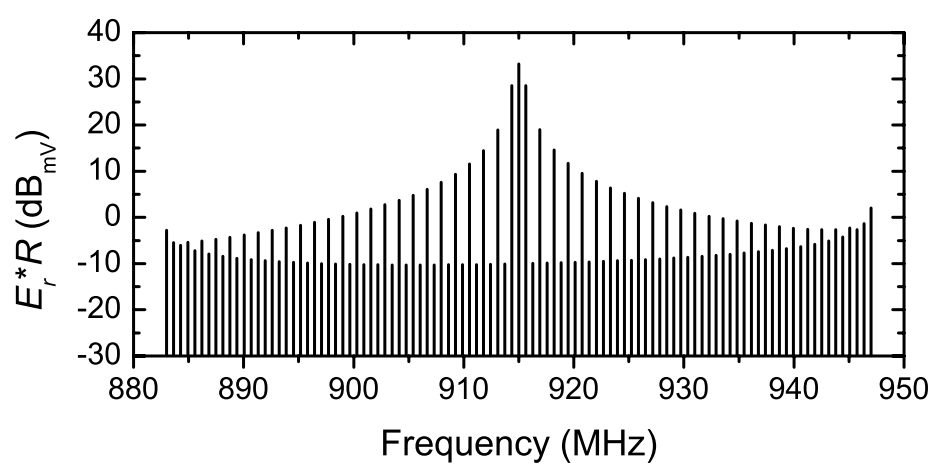

(a)

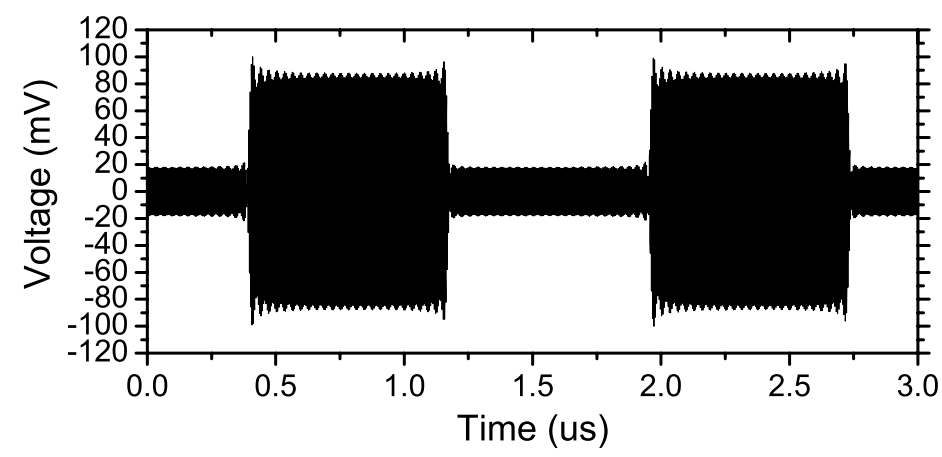

(b)

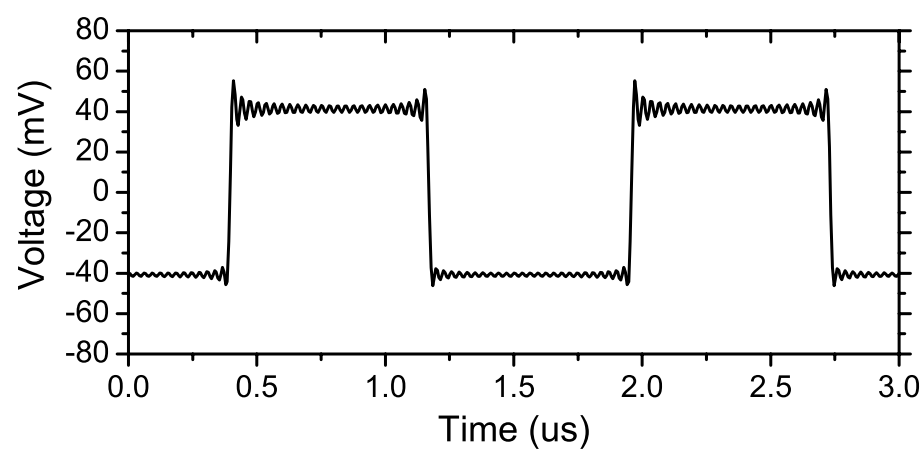

(c)

Fig. 3. Scattered field of linear wire antenna with capacitive time-varying load represented in (a) spectrum, (b) time-domain waveform, and (c) coherent demodulation waveform.

vector respectively, while $\left[V^{s r c}\right]$ is the unit-source vector due to the load [9]. $T$ is the transpose operation of the vector; $R$ is the distance from the loaded antenna to the observed point.

\section{Simulation results}

The simulation study is set as a linear wire antenna with length of $15.41 \mathrm{~cm}$ and radius of $1.541 \mathrm{~mm}$ operating at $915 \mathrm{MHz}$. The loading components are resistor changed in $10 \Omega / 1 \mathrm{~K} \Omega$ or capacitor changed in $0.1 \mathrm{pF} / 10 \mathrm{pF}$ with 
$640 \mathrm{KHz}$ square-wave waveform, which follows the data rate of the RFID standard EPC Gen. 2 [10]. The incident field is a uniform plane wave in $1.0 \mathrm{~V} / \mathrm{m}$ field strength.

The simulation results on the time-varying resistive load are shown in Fig. 2 including the frequency spectrum, the passband and the coherent detection time domain waveforms of the backscattering field $E_{r}$ multiplying the distance $R$. Note that the infinite Fourier series is truncated to a finite number of 100 in all of the computations. Meanwhile, the time-varying capacitive load simulation results are shown in Fig. 3. One can find that the capacitive load has lager amplitudes for the modulation waveforms, resulting easier demodulation for RFID readers. This information is useful for the RFID tag designs. Besides, the shown method provides a convenient way to fine-tune the loading impedance for the optimal system performance.

In practical situation, the loading impedance for the antenna is implemented by the integrated circuits, usually in CMOS technology. The simplest method for two-state resistive load is a NMOS FET with the control voltage via the gate port [11], where the resistance ratio for the ON/OFF state can reach from a few tens to a hundred as the numerical example shown. On the other hand, the varying capacitor in CMOS technology can be accomplished by a FET with source-drain connection to form a two-terminal device [11], which the gate dc voltage changes the capacitance dramatically from zero to a voltage over the FET threshold voltage. Typically the capacitance ratio more than a hundred is easy to achieve, resulting more apparent discrimination for the two logic states, thereby improving the receiving sensitivity of RFID readers.

\section{Conclusions}

This letter showed the theoretical analysis and numerical results of the backscattering modulation of the RFID system where the problem is treated as a time-varying loaded antenna using the equivalent circuit formulation with moment methods and the conversion matrix technique. Through the simulation studies, the capacitive load is more efficient than the resistive load for the receiver demodulation of the RDIF reader. The shown method and the associated results are useful for antenna and IC designs of RFID tags. 